\title{
Penile lymphoma presenting as an advanced penile cancer: report of a case
}

\author{
Konstantinos Stamatiou ${ }^{1}$, Hippocrates Moschouris ${ }^{2}$, Athanasios Marinis ${ }^{3 *}$, Drosos Karageorgopoulos ${ }^{4}$, Kassiani Manoloudaki ${ }^{5}$ \\ Correspondence: drmarinis@gmail.com \\ ${ }^{1}$ Department of Urology, ${ }^{2}$ Department of Radiology, ${ }^{3}$ First Department of Surgery, ${ }^{4}$ Department of Internal Medicine and \\ ${ }^{5}$ Department of Pathology, Tzaneio General Hospital, Zanni \& 1 Afentouli, GR18536, Piraeus, Greece.
}

\begin{abstract}
Penile lymphoma is a very rare neoplasm. We report the case of an 82 -year-old man who presented with phimosis. The patient also complained of frequent and painful urination. Upon examination a painless penile ulcer and multiple inguinal lymph nodes were found. The shaft of the penis and the prostate were hard on palpation. Abdominal and transrectal ultrasound examination confirmed the involvement of the penis shaft and the prostate and also revealed involvement of the urinary bladder. Biopsy showed diffuse, large B-cell lymphoma. The patient was treated with systemic chemotherapy with full remission of the disease. We review the literature relevant to penile lymphoma and discuss this uncommon condition.
\end{abstract}

Keywords: Genital ulcer, male urogenital diseases, lymphoma, non-hodgkin, penile neoplasms, large b-cell, diffuse

\section{Background}

Penile malignant tumors are generally uncommon. The most common type, squamous carcinoma, accounts for less than $1 \%$ of all malignancies in men in the USA and for $0.1 \%$ of cancer deaths [1]. Although non-Hodgkin's lymphoma occurs at extranodal sites in up to $48 \%$ of patients [2], lymphomas of the penis are rare and in most cases represent secondary involvement of the penis by lymphoma due to haematogenous or lymphatic spread or due to direct infiltration from a neighbouring organ [3]. Thus, primary penile lymphoma is extremely rare and indeed, less than 30 cases have been reported in the literature since Oomura et al first described the entity in 1962 [4].

Given its rarity, it is unusual for physicians to consider lymphoma in the differential diagnosis of a penile mass.

\section{Case presentation}

An 82 year-old man presented with phimosis. He also complained of frequent and painful urination. He had a history of chronic hepatitis $C$. His physical examination revealed a large welldemarcated penile ulcer with a necrotic base (Figure 1). The shaft of the penis and the prostate were hard on palpation. Multiple inguinal lymph nodes and mild abdominal tenderness were also noted. No urethral discharge, scrotum abnormalities, or hepatosplenomegaly was noted. Full blood count results were as follows: Hct: 38,1\%, WBC:6980/ $\mu$ l (Neu 65\%, Lym 26\%), PLT:250000/ $\mu \mathrm{l}$. Serum biochemistry tests were as follows: Glu: 119 mg/dl, Ur: 30 mg/dL, Cr: 0,9 mg/dL, CPK:51 U/l, LDH:276
U/I. Serum electrolytes were normal. Serum PSA was 0,52ng/ $\mathrm{ml}$. Abdominal ultrasound (US) showed a normal shape and configuration of both kidneys. Irregular thickening of the urinary bladder was noted. The prostate was enlarged (volume: $80 \mathrm{ml})$, hypoechoic and with indistinct borders. Transrectal US examination showed that the shaft of the penis and the right prostate lobe were infiltrated by a solid, ill-defined, hypoechoic mass (Figure 2a). Multiple irregular solid masses were also found to protrude into the lumen of the bladder. Computed tomography (CT) of the abdomen and pelvis showed pelvic lymphadenopathy and significant prostate enlargement but failed to provide further information. CT of the chest showed no metastases to the lungs or thoracic lymphadenopathy. Magnetic resonance imaging (MRI) of the upper and lower abdomen showed a tumor in the location of the seminal vesicles and prostate of nodular/tubular inhomogeneous composition exhibiting little contrast enhancement, as well as thickening of the bladder wall and a filling defect between 5 o'clock and 9 o'clock, probably due to arteriovenous/lymphatic dysplasia.

The remaining abdominal organs and lymph nodes revealed no pathological findings. Fine needle aspiration of one of the inguinal masses and biopsy of the penile ulcer and bladder tumor were performed. The histopathologic examination showed that the tissue samples from the penis were covered focally by stratified squamous epithelium and exhibited surface ulceration. The underlying layers were invaded to a considerable extent by a malignant neoplasm of lymphatic origin with

(c) 2012 Marinis et al; licensee Herbert Publications Ltd. This is an open access article distributed under the terms of Creative Commons Attribution License (http://creativecommons.org/licenses/by/3.0), This permits unrestricted use, distribution, and reproduction in any medium, provided the original work is properly cited. 


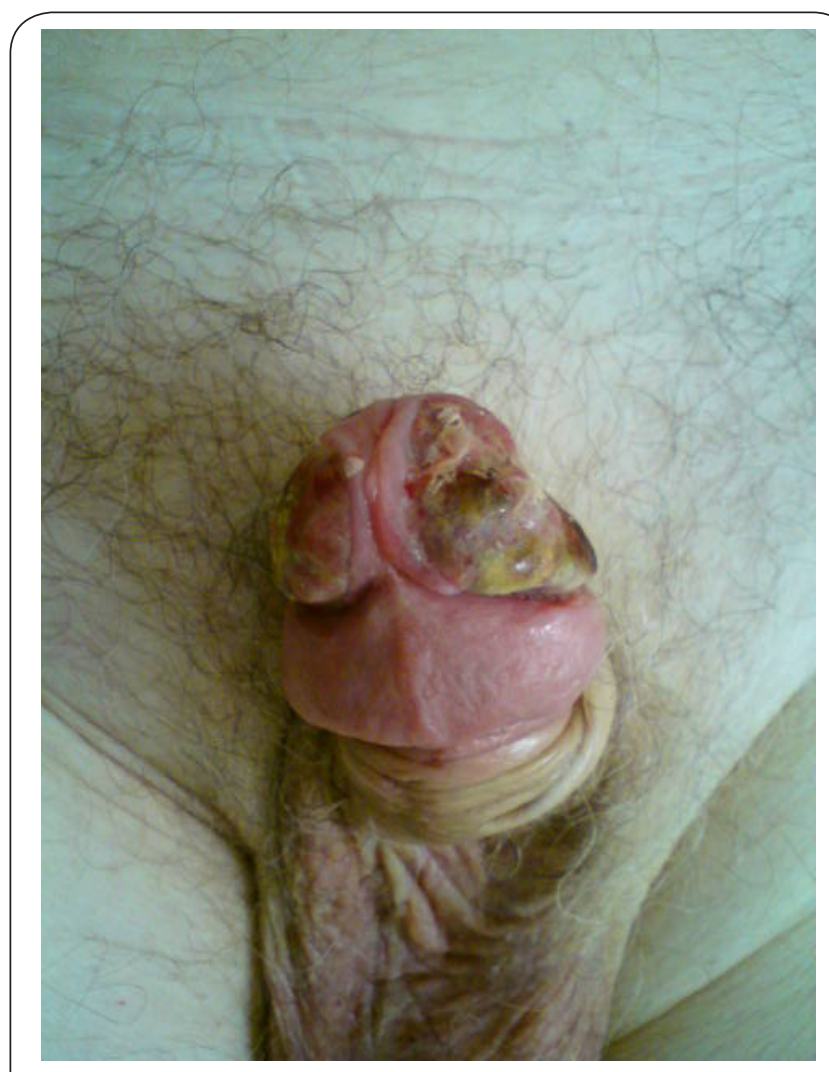

Figure 1. Dorsal view of the necrotic ulcer on the glans penis.

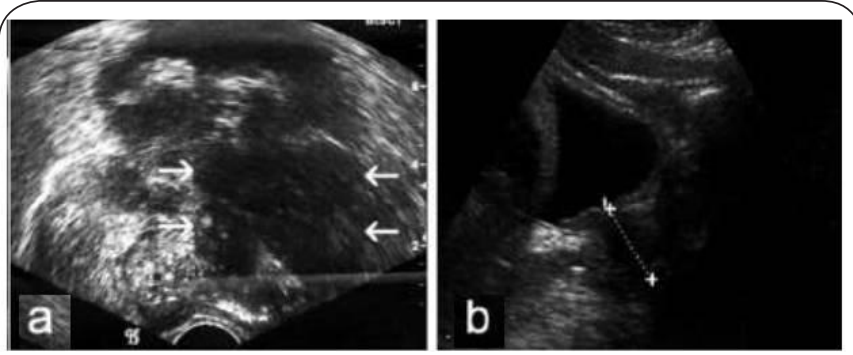

Figure 2. Sonographic imaging of the case.

a: Transrectal US confirms infiltration of the prostate by a bulky, lobulated, hypoechoic mass (arrows) which also involves the base of the penis.

b: Transabdominal US shows resolution of the infiltrative process.

morphological and immunohistochemical characteristics of a diffuse lymphoma of large cell (centroblast) B type with a high malignant potential (Figure 3). The immunophenotyping showed positive staining, diffusely for LCA, CD19, CD20, CD79a and focally for T, CD3, CD5, CD68, and no staining for epithelial neuroendocrine markers or melanoma-specific markers. A fine needle aspiration of one of the inguinal masses and biopsy of the bladder also revealed atypical lymphocytes (B-cells) consistent with diffuse large B-cell lymphoma. Staining was

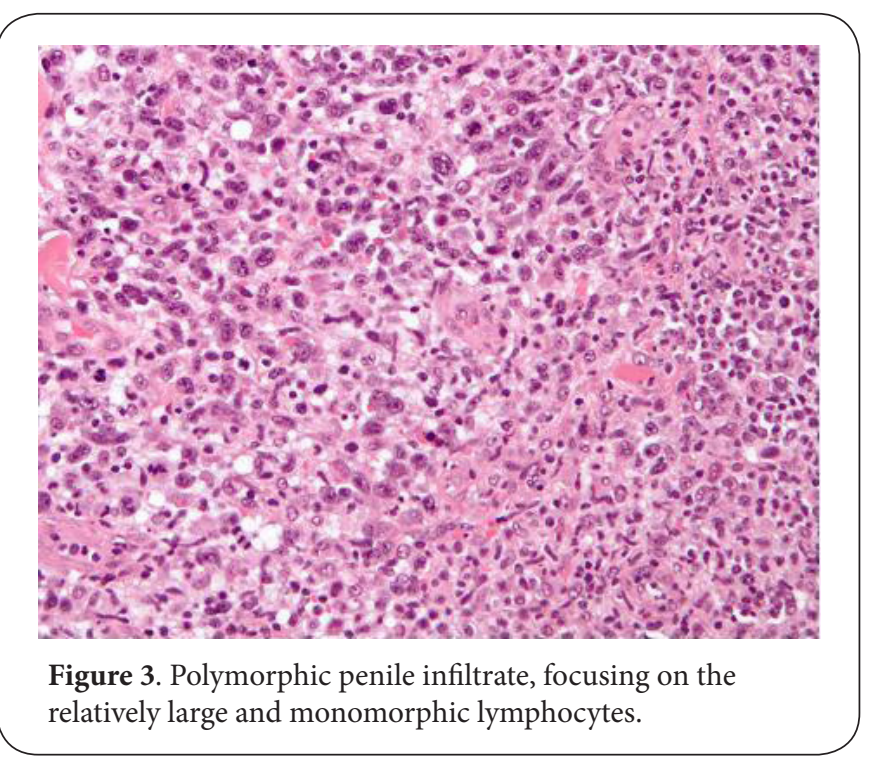

negative for acid-fast bacilli, while culture for bacteria and fungi did not yield any growth. The bone marrow biopsy did not show any infiltration by lymphoma. The patient was treated with systemic chemotherapy. Six cycles of CHOP (Cyclophosphamide, Hydroxydaunorubicin, Oncovin, Prednisone) were given resulting in healing of the penile ulcer and resolution of the inguinal lymphadenopathy. In the post-treatment transabdominal US the bladder and prostate lesions also had completely resolved (Figure $\mathbf{2 b}$ ).

\section{Discussion}

Primary penile lymphoma is extremely rare and in most of the reported cases it is located in the shaft of the penis and/ or the glans penis [5]. Diffuse large cell lymphoma is the most common histological subtype [6]. Both B-cell and T-cell subtypes have been reported [7]. The clinical presentation of penile lymphoma includes indurated plaques, nodules, diffuse penile swelling and ulceration with or without induration [6]. Rare associated symptoms include phimosis, priapism, lymphadenopathy and testicular enlargement [9]. In most of the reported cases the disease has reached an advanced stage at the time of diagnosis [3]. The diagnosis of penile lymphoma can be demanding. A major differential diagnostic challenge is the distinction between this entity and the squamous cell carcinoma of the penis. In fact, failure to recognize lymphoma may result in unnecessary penile amputation, which is a common treatment option for squamous cell carcinoma and other malignant primary tumours of the penis, especially when the tumor involves the entire shaft. Therefore, a full physical examination and an excisional biopsy are essential to reach the correct diagnosis. The differential diagnosis also includes vasculitis, trauma, and sexually transmitted diseases [10]. The workup should include serological and microbiological studies for sexually transmitted diseases, 
especially when the tumor presents as ulceration or with other inflammatory signs mimicking an infection. Since staging and differentiation between primary lymphoma and disease secondary to systemic lymphoma are important in terms of treatment and prognosis, systemic radiological investigation, including $\mathrm{CT}$, MRI or positron emission tomography scan, should be undertaken [3]. While systemic chemotherapy is the treatment of choice for a secondary presentation of lymphoma in the penis, in primary penile lymphoma, treatment guidelines are difficult to establish, because of the very limited number of cases. Recommendations have included chemotherapy, local radiotherapy, surgery, or combined modalities of both surgery and chemotherapy or radiotherapy and chemotherapy [4-10]. Whether to use one, two, or all of these modalities to eradicate the tumour, while attempting to preserve the anatomy and physiology of the penis, is controversial. On reviewing the literature, it appears that the management of primary penile lymphoma depends mainly on the stage of the disease, as well as the age and performance status of the patient. Since malignant lymphoma is a systemic disease that can metastasize haematogenously, and treatment by chemotherapy and radiotherapy allow preservation of function of the penis, radical surgery appears to be contraindicated and should only be used after failure of other modalities [8]. However, penile lymphoma is a disease of the elderly and preservation of the penis may have little importance for many such patients. Given that chemotherapy in the elderly results in significant morbidity, surgery may be a good treatment option in patients for whom chemotherapy is not appropriate. Radiotherapy alone offers a potential cure rate of $65 \%$ in advanced stage lymphoma and thus could be considered as a valuable treatment option [3]. However, similar to surgery, radiotherapy may result in disfigurement or loss of erectile function and thus it may be avoided in younger patients. Moreover, there is no consensus as to the dose of radiotherapy and most importantly radiotherapy cannot eliminate occult disseminated disease. Systemic chemotherapy is a good treatment option because it preserves the erectile function and avoids disfigurement. The reported 2-year disease-free survival after chemotherapy has been as high as $83 \%$ for primary diffuse large cell lymphoma [11]. The usual chemotherapy regimen is $\mathrm{CHOP}$, however other regimens have also been used, such as rituximab-containing ones [9]. To our knowledge, good outcomes with complete remission and preservation of penile function with chemotherapy alone have been documented after the completion of a second cycle of chemotherapy [11]. This is of utmost importance given the morbidity that can be associated with this treatment modality. Combined modality treatment seems to be the ideal treatment option, although toxicity can restrict the use of chemotherapy [12].

\section{Conclusions}

We report the case of an elderly patient with a penile ulcer that was associated with a diffuse large B-cell lymphoma infiltrating the adjacent organs. The tumour resolved completely after systemic chemotherapy with the $\mathrm{CHOP}$ regimen. A high index of suspicion is required to correctly identify this rare entity and to institute appropriate treatment avoiding potentially unnecessary mutilating surgery.

\section{Competing interests}

All authors declare that they do not have any financial or other competing interests.

\section{Author contributions}

All authors had substantial contributions to conception and design, acquisition of data, or analysis and interpretation of data; Stamatiou $\mathrm{K}$ and Moschouris $\mathrm{H}$ equally contributed in drafting the article; Marinis A and Karageorgopoulos $D$ revised it critically for important intellectual content; Manoloudaki K drafted the pathology section and, all authors gave final approval of the version to be published.

Publication history

Received: 1-Aug-2012 Revised: 3-Aug-2012

Accepted: 3-Aug-2012 Published: 16-Aug-2012

\section{References}

1. Schellhammer P, Jordan GH, Schlossberg SM. Tumors of the penis. In: Walsh PC, Retik AB, Stamey TA, Vaughn ED, editors. Campbell's Urology. 6th ed. Philadelphia: W. B. Saunders Co; 1992. p. 1264-91.

2. Zucca E, Gregorini A, Cavalli F: Management of non-Hodgkin lymphomas arising at extranodal sites. Ther Umsch 2010; 67;(10.);51725. | PubMed

3. Chiang $\mathrm{KH}$, Chang PY, Lee SK, Yen PS, Ling $\mathrm{CM}$, Lin CC, et al.: MR findings of penile lymphoma. Br J Radiol 2006; 79;(942.);526-8. | Article | PubMed

4. McNab PM, Jukic DM, Mills O, Browarsky I: Primary cutaneous CD30+ T-cell lymphoproliferative disorder presenting as paraphimosis: a case report and review of the literature. Dermatol Online J 2011; 17;(7.);3. | Article | PubMed

5. Clemens AJ, Thiel DD, Wu KJ: Primary cutaneous T cell lymphoma of the external genitalia. J Urol 2011; 185;(2.);687-8. | Article | PubMed

6. Arena F, di Stefano C, Peracchia G, Barbieri A, Cortellini P: Primary lymphoma of the penis: diagnosis and treatment. Eur Urol 2001; 39;(2.);232-5. PubMed

7. Pomara G, Cuttano MG, Tripodo C, Carlino F, Selli C: Primary T-cell rich B-cell lymphoma of the penis: a first case. BJU Int 2003; 91;(9.);889. I Article | PubMed

8. Jabr Fl: Recurrent lymphoma presenting as a penile ulcer in a patient with AIDS. Dermatol Online J 2005; 11;(2.);29. I Article I PubMed

9. Hamamoto S, Tozawa K, Nishio H, Kawai N, Kohri K: Successful treatment of primary malignant lymphoma of the penis by organpreserving rituximab-containing chemotherapy. Int J Clin Oncol 2012; 17;(2.);181-4. | Article | PubMed

10. Lin DW, Thorning DR, Krieger JN: Primary penile lymphoma: diagnostic difficulties and management options. Urology 1999; 54;(2.);366. I 
Marinis et al. Journal of Cancer Therapeutics \& Research 2012, http://www.hoajonline.com/journals/pdf/2049-7962-1-21.pdf

Article | PubMed

11. Tomb RR, Stephan F, Klein-Tomb L, Chahine G, Grosshans E: Recurrent primary CD30+ lymphoma of the penis. Br J Dermatol 2003;

149;(4.);903-5. I Article I PubMed

12. El-Sharkawi A, Murphy J: Primary penile lymphoma: the case for combined modality therapy. Clin Oncol (R Coll Radiol) 1996; 8;(5.);334-

5. | Article | PubMed

\section{Citation:}

Stamatiou K, Moschouris H, Marinis A, Karageorgopoulos D and Manoloudaki K:

Penile lymphoma presenting as an advanced penile cancer: report of a case. journal of Cancer Therapeutics and Research 2012, 1:21. http://dx.doi.org/10.7243/2049-7962-1-21 